\title{
Nonalcoholic fatty liver disease and fatigue in long-term survivors
}

of childhood-onset craniopharyngioma

A Hoffmann ${ }^{1}, K_{\text {Bootsveld }}^{2}$, U Gebhardt ${ }^{1}$, AMM Daubenbüchel ${ }^{1,3}$, AS Sterkenburg ${ }^{1,3}$, HL Müller $^{1}$

${ }^{1}$ Department of Pediatrics, Klinikum Oldenburg, Medical Campus University Oldenburg; ${ }^{2}$ Radiologie Oldenburg, Oldenburg, Germany;

${ }^{3}$ University of Groningen, The Netherlands. www.kraniopharyngeom.net

\section{Introduction}

Hypothalamic obesity in childhood craniopharyngioma (CP) carries a high risk for development of metabolic syndrome. In metabolic syndrome, the development of non alcoholic fatty liver disease (NAFLD) is known. The aim of this study is to detect the risk for NAFLD in childhood-onset CP.

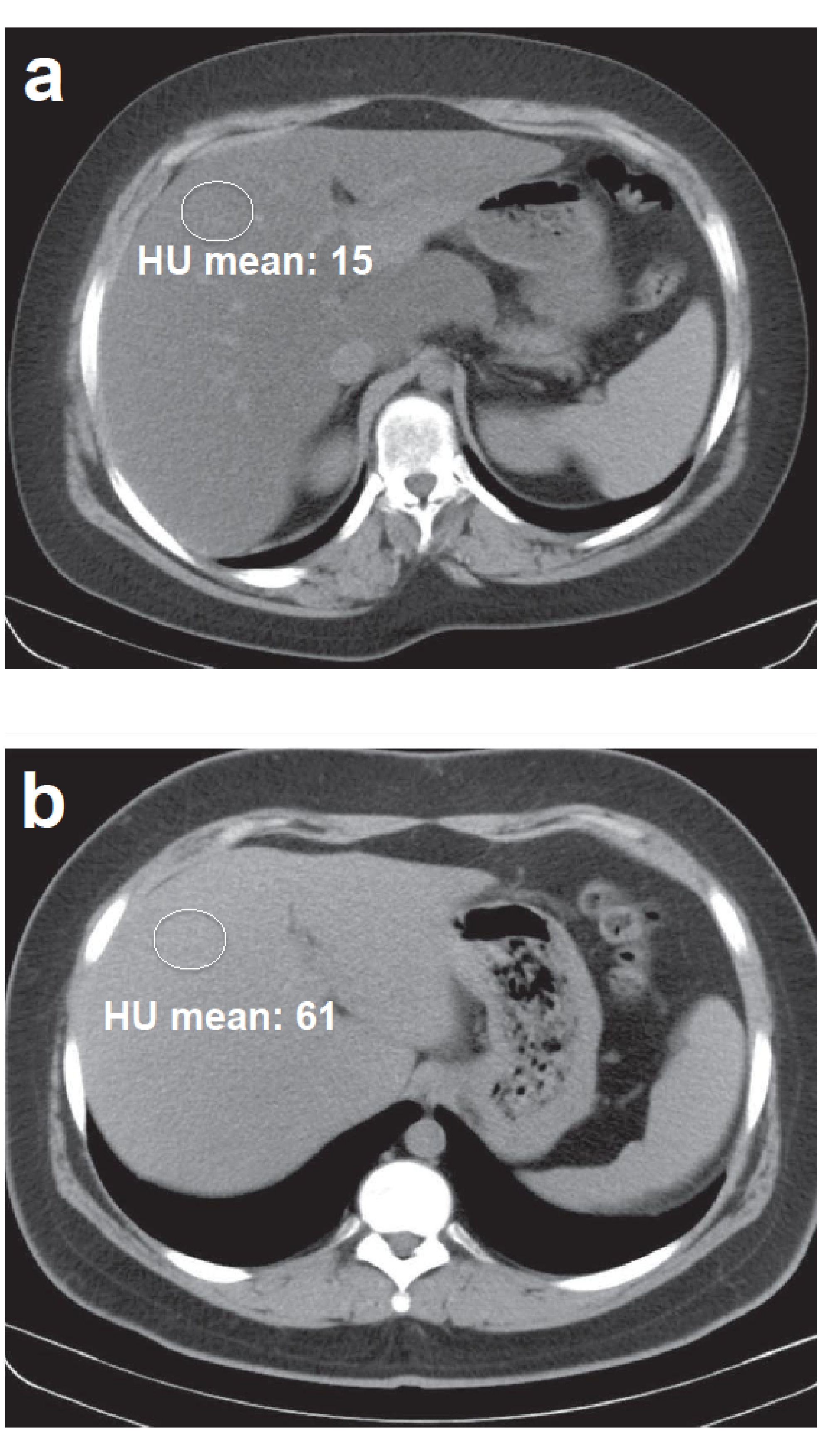

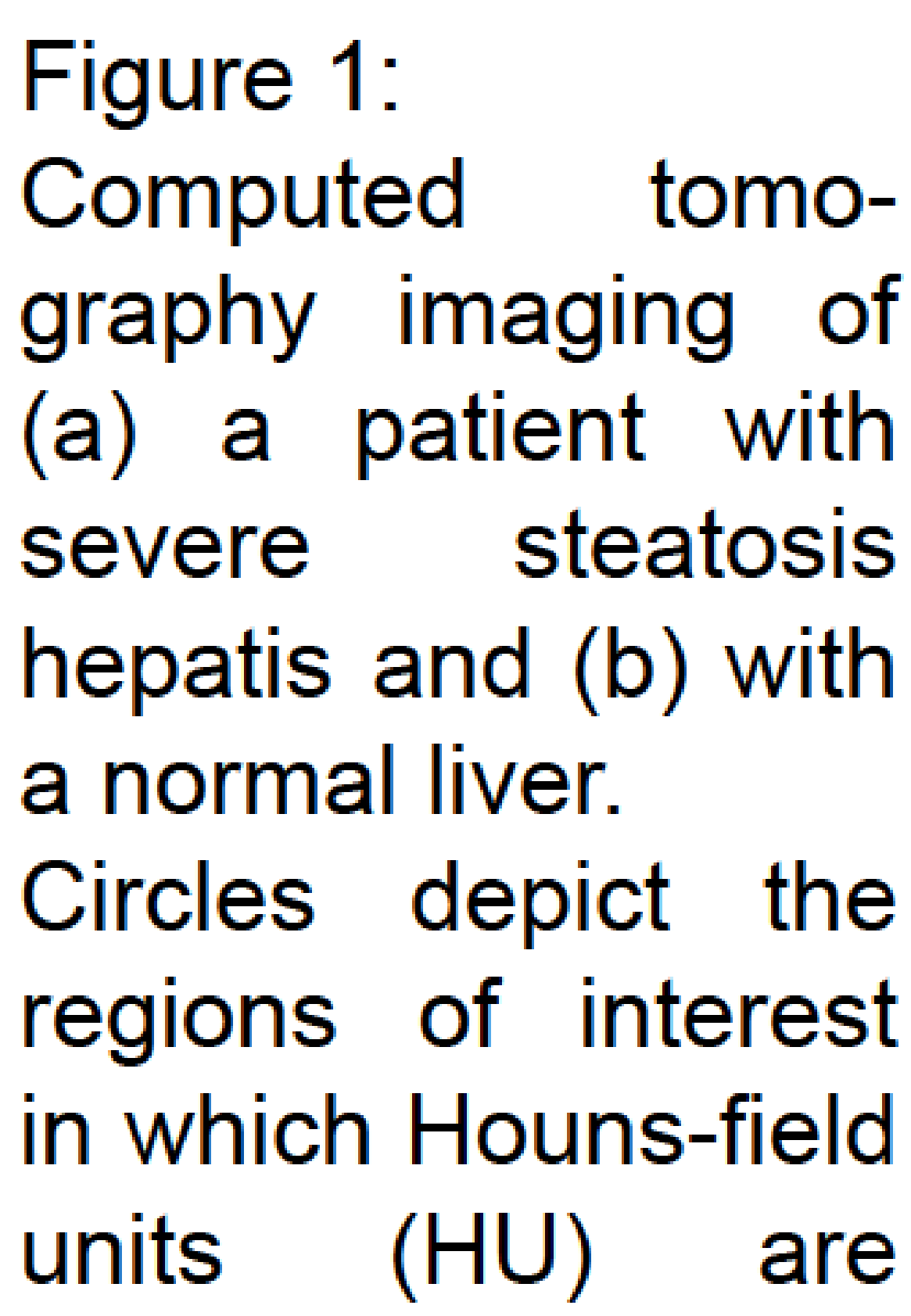
measured.

Table 1: Characteristics of 19 patients with $\mathrm{CP}$ at time of diagnosis and current study. BMI SDS was calculated according to Rolland-Cachera et al. Bold-formatting indicates significant differences.

\section{Patients and Methods}

A total of 384 patients recruited in trials HIT Endo and KRANIOPHARYNGEOM 2000 were analyzed. Ninety-four survivors were included by fulfilling the criteria of proven hypothalamic involvement $(\mathrm{HI})$, a minimum interval of 5 years between diagnosis and study, and a minimum age of 18 years at time of evaluation. 19 patients participated.

\begin{tabular}{|c|c|c|c|}
\hline Characteristics & $\begin{array}{l}\text { Patients without } \\
\text { steatosis hepatis }\end{array}$ & $\begin{array}{l}\text { Patients with } \\
\text { steatosis hepatis }\end{array}$ & $\mathbf{P}$ \\
\hline Number of patients $(n)$ & 9 & 10 & \\
\hline Age at diagnosis (years), median (range) & $11.9(2.8-20.5)$ & $9.39(5.06-19.6)$ & 0.859 \\
\hline Age at study (years), median (range) & $23.7(18.9-29.2)$ & $25.16(16.9-30.3)$ & 0.591 \\
\hline Follow-up (years), median (range) & $12.8(6.0-24.1)$ & $14.7(6.1-19.1)$ & 0.765 \\
\hline Gender, $\mathrm{n}$ (male/female) & $4 / 5$ & $5 / 5$ & \\
\hline $\begin{array}{l}\text { Degree of resection, } \mathrm{n}(\%) \\
\text { Complete resection } \\
\text { Incomplete resection }\end{array}$ & $\begin{array}{l}3(33.4) \\
6(66.7)\end{array}$ & $\begin{array}{l}5(50) \\
5(50)\end{array}$ & \\
\hline Hypothalamic involvement, n (\%) & $9(100)$ & $10(100)$ & \\
\hline BMI-SDS at diagnose, median (range) & $0.66(\mathrm{~K} 0.6-4.67)$ & $0,70(\mathrm{~K} 1.6-3.65)$ & 0.530 \\
\hline BMI-SDS at study, median (range) & $5.48(1.51-10.29)$ & $7.30(3.71-12.62)$ & 0.331 \\
\hline $\begin{array}{l}\text { Body composition, \%, median (range) } \\
\text { Body fluid } \\
\text { Body fat }\end{array}$ & $\begin{array}{l}49.9(41.0-56.7) \\
35.4(23.9-49.3)\end{array}$ & $\begin{array}{l}45.3(36.7-51.6) \\
42.6(32.9-55.8)\end{array}$ & $\begin{array}{l}0.036 \\
0.033\end{array}$ \\
\hline Hounsfield units (HU), median (range) & $57(46-63)$ & $32(15-41)$ & \\
\hline
\end{tabular}

\begin{tabular}{|c|c|c|c|}
\hline Serum parameters & $\begin{array}{l}\text { Patients without } \\
\text { steatosis hepatis } \\
\text { n, median (range) }\end{array}$ & $\begin{array}{c}\text { Patients with steatosis } \\
\text { hepatis } \\
\text { n, median (range) }\end{array}$ & $\mathbf{P}$ \\
\hline Cholesterin (mg/dl) & $8 ; 177(144-263)$ & $10 ; 199(150-271)$ & 0.479 \\
\hline $\mathrm{HDL}$ (mg/dl) & $8 ; 40(26-58)$ & $10 ; 34(16-52)$ & 0.169 \\
\hline $\mathrm{LDL}(\mathrm{mg} / \mathrm{dl})$ & $8 ; 119(86-213)$ & $10 ; 147(59-218)$ & 0.818 \\
\hline AST (U/I) & $9 ; 29(21-39)$ & $10 ; 55(18-126)$ & 0.041 \\
\hline $\mathrm{ALT}(\mathrm{U} / \mathrm{l})$ & $9 ; 28(13-62)$ & $10 ; 47(18-232)$ & 0.141 \\
\hline GLDH (U/I) & $9 ; 2.95(0.3-7)$ & $10 ; 13.4(3.2-26.7)$ & 0.006 \\
\hline ChE (U/l) & $9 ; 9.95(7-11.9)$ & $10 ; 9.8(6.6-10.9)$ & 0.701 \\
\hline Gamma-GT (U/I) & $8 ; 23(7.8-99)$ & $10 ; 94(18-216)$ & 0.016 \\
\hline Billirubin (mg/dl) & $9 ; 0.55(0.2-1.7)$ & $10 ; 0.4(0.09-1.5)$ & 0.428 \\
\hline Insulin (mU/l) & $9 ; 17.15(2.3-36.2)$ & $9 ; 47.2(13.0-58.8)$ & 0.03 \\
\hline $\mathrm{HbA} 1 \mathrm{c}(\%$ of $\mathrm{Hb})$ & $9 ; 5.2(4.5-7.0)$ & $10 ; 5.8(5.1-6.3)$ & 0.408 \\
\hline Blood glucose $(\mathrm{mg} / \mathrm{dl})$ & $9 ; 75(58-101)$ & $10 ; 79(58-115)$ & 0.429 \\
\hline HOMA & $8 ; 2.45(0.3-5.5)$ & $5 ; 9.2(2.2-14.8)$ & 0.036 \\
\hline
\end{tabular}

Table 2: Serum parameters of 19 patients $\mathrm{CP}$ at time of study. Bold-formatting indicates significant differences. HOMA: homeostasis model assessment index

\begin{tabular}{l|c|c|c|c|}
\hline Medication & \multicolumn{2}{c|}{$\begin{array}{c}\text { Patients without } \\
\text { steatosis hepatis }\end{array}$} & \multicolumn{2}{c|}{$\begin{array}{c}\text { Patients with } \\
\text { steatosis hepatis }\end{array}$} \\
\hline & $\mathrm{n}$ & $\%$ & $\mathrm{n}$ & $\%$ \\
\hline Cortisone & 8 & 89 & 10 & 100 \\
DDAVP & 9 & 100 & 10 & 100 \\
L thyroxine & 8 & 89 & 10 & 100 \\
*Rec. GH & 6 & 67 & 6 & 60 \\
Sex steroids & 8 & 89 & 10 & 100 \\
Methylphenidate & 0 & 0 & 5 & 50 \\
Melatonin & 4 & 44 & 0 & 0 \\
Anti-diabetics & 0 & 0 & 1 & 10 \\
Cardiac medicine & 0 & 0 & 2 & 20 \\
\hline Table 3: Medication & of the 19 & patients with childhood-onset
\end{tabular}

Table 3: Medication of the 19 patients with childhood-onset craniopharyngioma (CP) at the time of current study.

\begin{tabular}{|l|c|c|c|c|}
\hline Medication & \multicolumn{2}{c|}{$\begin{array}{c}\text { Patients without } \\
\text { steatosis hepatis }\end{array}$} & \multicolumn{2}{c|}{$\begin{array}{c}\text { Patients with } \\
\text { steatosis hepatis }\end{array}$} \\
\hline & $\mathrm{n}$ & $\%$ & $\mathrm{n}$ & $\%$ \\
\hline Cortisone & 8 & 89 & 10 & 100 \\
\hline DDAVP & 9 & 100 & 10 & 100 \\
L thyroxine & 8 & 89 & 10 & 100 \\
*Rec. GH & 4 & 44 & 6 & 60 \\
Sex steroids & 8 & 89 & 10 & 100 \\
Methylphenidate & 0 & 0 & 6 & 60 \\
Melatonin & 2 & 22 & 1 & 10 \\
Anti-diabetics & 1 & 11 & 4 & 40 \\
Cardiac medicine & 3 & 33 & 2 & 20 \\
\hline
\end{tabular}

Table 4: Medication of 19 pts with childhood-onset craniopharyngioma during follow-up 2-7 yrs after original study.

\section{Results}

NAFLD occurs in about $50 \%$ of CP patients with $\mathrm{HI}$ and is associated with elevated liver enzymes and homeostasis model assessment index. BMI is not an effective predictive factor but body fat mass measured by near-infrared spectroscopy (NIRS) is. Over half of CP patients $(60 \%)$ with NAFLD are treated with stimulating agents.

\section{Conclusions}

NAFLD is a major adverse late effect in childhood-onset CP. NIRS rather than BMI should be used to measure body composition and predict NAFLD. Stimulating agents for treatment of fatigue and daytime sleepiness in CP should be prescribed judiciously.

Supported by German Childhood Cancer Foundation 